\title{
Joint Scheduling and Relay Selection in One- and Two-Way Relay Networks with Buffering
}

\author{
Lianghui Ding, Meixia Tao, Fan Yang, Wenjun Zhang \\ Department of Electronic Engineering \\ Shanghai Jiao Tong University, China \\ Email: \{lhding, mxtao, 46265878, zhangwenjun\}@sjtu.edu.cn
}

\begin{abstract}
In most wireless relay networks, the source and relay nodes transmit successively via fixed time division (FTD) and each relay forwards a packet immediately upon receiving. In this paper we enable the buffering capability of relay nodes and propose a framework for joint scheduling and relay selection. The goal is to maximize the system long-term throughput by fully exploiting multi-user diversity in the network. We develop two joint scheduling and relay selection (JSRS) algorithms for unidirectional and bidirectional traffic, respectively. The novel cross-layer relay selection metrics which our algorithms are based upon take into account both instantaneous channel conditions and the queuing status. We also demonstrate that the proposed JSRS can be realized in a distributed way without explicit coordination among the network nodes. Extensive simulation is carried out to evaluate the performance of the proposed JSRS with buffering in comparison with traditional FTD without buffering. Typical throughput enhancements up to $101 \%$ and $110 \%$ are observed in one-way and two-way relay networks respectively, at low signal-to-noise ratio $(0 \mathrm{~dB})$.
\end{abstract}

\section{INTRODUCTION}

Relay transmission can enhance the reliability, increase the rate and save the power in wireless networks [1], and thus has received considerable attention from researchers recently [2][10]. The two popular relay strategies are known as decodeand-forward (DF) and amplify-and-forward (AF) [1]. In a multiple-relay network, relay selection is a promising technique to improve the system performance. Relay selection for minimum packet error probability is analyzed for DF in [2], for AF in [3] and for hybrid DF and AF in [4]. Relay selection for minimum outage probability under an aggregate power constraint is analyzed in [5]. Authors in [5], [6] also presented a timer-based distributed relay selection algorithm which requires no topology information.

Currently, two-way relaying is shown to be able to significantly reduce the spectral loss caused by the half-duplex constraint in practical systems when compared with unidirectional relaying [7]. There are also DF and AF modes for two-way relay networks, in which the AF relay strategy is similar to that in one-way relay network, while the DF applies the concept of network coding. One popular two-way DF strategy is to let the relay node perform bit-level XOR operation on the information packets received from the two source nodes and then broadcast the new packet to the two

This work is supported in part by Shanghai Educational Development Foundation (No. 2008CG14) and in part by Natural Science Foundation of Shanghai (No. 08ZR1410400). desired receivers simultaneously [8]-[10]. The conventional relay selection methods proposed for unidirectional traffic may be applied to the bidirectional communication scenario after some modifications. In [11], a bidirectional relay selection criterion is proposed, wherein the relay is chosen to maximize the weighted sum of the bidirectional rate pair on the boundary of the achievable rate region.

In the aforementioned research on both one-way and twoway relay networks, it is always assumed that the source and relay nodes transmit in successive time slots. In other words, the relay node forwards the received packets immediately (with or without processing the signal) and there is no need for the relays to buffer them. We refer to this kind of scheduling method as fixed time division (FTD). If, however, the endto-end delay requirement of the traffic is not stringent, the scheduled transmission between the source and relay nodes with respect to the instantaneous channel condition can be exploited to obtain multi-user diversity and hence improve the long-term throughput of the system. This requires the relay nodes to have buffer to store the received packets. So far, to our best knowledge, little work has considered the scheduling issue among source nodes and relay nodes in relay networks.

In this paper, we consider the joint scheduling and relay selection in relay networks for both one-way and two-way communications. Each relay is assumed to have enough (but not infinite) buffer size. We propose a cross-layer relay selection metric that takes into account both the instantaneous channel condition and the queuing status. Using this metric, we then schedule the transmission between the source nodes and relay nodes. Simulation results show that the system throughput is improved significantly compared with traditional relay selection using fixed time division (FTD) scheme.

The rest of the paper is organized as follows: In Section II, we present the system model of one- and two-way relay networks and discuss the optimal relay selection for FTD scheme. Section III, we propose our joint scheduling and relay selection (JSRS) algorithms. Simulation results are given in Section IV. Finally, we conclude the paper in Section V.

\section{Network Model and Relay Selection without BUFFERING}

In this section, we introduce the network model and disucss the optimal relay selection criteria of the fixed time division 
(FTD) based scheduling in both one-way and two-way relay networks.

\section{A. Network Model}

As shown in Figure 1, we consider a relay network that consists of a source node $s$, a destination node $d$, and $K$ relay nodes. For bidirectional traffic, the destination node $d$ is also a source node, and node $s$ is also a destination node. Thus, instead of calling them as source or destination nodes, we simply mention them as node $s$ or node $d$ in the case of bidirectional traffic. There are two buffers in each relay, $B_{k}^{s}$ and $B_{k}^{d}$, for packets received from nodes $s$ and $d$, respectively. In the case of unidirectional traffic, only $B_{k}^{s}$ is needed. The queue sizes of $B_{k}^{s}$ and $B_{k}^{d}$ are denoted as $Q_{k}^{s}$ and $Q_{k}^{d}$ respectively.

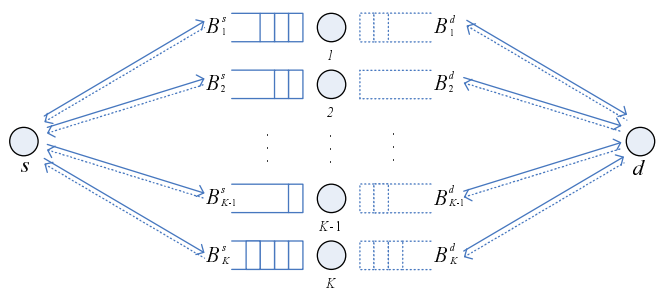

Fig. 1. Network model of one- and two-way relaying with buffering.

Throughout this paper, we make following assumptions. The transmission is time slotted and packet-based [12]. In each time slot, a packet is transmitted with a rate determined by the current channel condition. The length of the time slot, or equivalently the transmission time of each packet, is adaptive with respect to the channel gain. The channel coefficients between any pair of nodes in the network are all independent. The channel coherence time of each link is much larger than the duration of each packet transmission, but much smaller than the delay requirement of the transmission application. There is no direct link between node $s$ and node $d$. Each relay adopts DF relaying strategy. In the case of one-way relaying, the relay node just re-encodes the detected packet from the source and transmits to the destination. For twoway relaying, the relay node performs bit-level XOR operation on the two detected packets from nodes $s$ and $d$, re-encodes the resulting new packet and then broadcasts it to $s$ and $d$ simultaneously. In addition, each transmitting node has the same and fixed transmission power, and the channel gains of forward and reverse links are symmetric. That means the maximum achievable rate from node $i$ to node $j$ is the same as that from node $j$ to node $i$, for $i, j \in\left\{s, d, r_{1}, \ldots, r_{K}\right\}$.

\section{B. Relay Selection without Buffering}

In this subsection, we present the relay selection criteria using the traditional FTD based scheduling. Let $C_{i j}$ denote the maximum achievable transmission rate in bits $/ \mathrm{sec} / \mathrm{Hz}$ of the link $l_{i j}$ from node $i$ to node $j$. It is a function of the instantaneous channel condition of the link and given by

$$
C_{i j}=\log \left(1+\gamma_{0} \alpha_{i j}\right),
$$

where $\gamma_{0}$ is the average received signal-to-noise ratio (SNR) and assumed to be same for the active links considered in this paper, and $\alpha_{i j}$ is the composite channel gain. The composite channel gain contains two components, the small scale normalized Rayleigh fading, and the shadowing that follows log-normal distribution.

To facilitate the XOR-based combining at each relay node and the XOR-based detection at each desired destination node in the case of bidirectional traffic, we also assume that all the packets are numbered and contain the same number of information bits. Without loss of generality, the length of each packet is normalized to one. Thus the transmission time of link $l_{i j}$ is $1 / C_{i j}$, which as a result depends on the channel gain $\alpha_{i j}$.

In one-way relay networks, suppose relay $k$ is selected to forward packets. Then, the end-to-end transmission time of a packet from the source $s$ to the destination $d$ is [13]

$$
t_{k}^{1 w}=\frac{1}{C_{s r_{k}}}+\frac{1}{C_{d r_{k}}} .
$$

Therefore, the optimal relay selection criterion is to select the relay node with the minimum transmission time.

In two-way relay networks, there are two XOR-based relay schemes, which are the 2-step-XOR and the 3-step-XOR [9]. For the 2-step-XOR, both nodes $s$ and $d$ transmit to relay nodes simultaneously in phase I, and the relay nodes forward the combined packet in phase II. For 3-step-XOR, nodes $s$ and $d$ send packets to relay $k$ sequentially in the first two phases. And the rates are $C_{s r_{k}}$ and $C_{d r_{k}}$ respectively. In the last phase, the relay decodes and combines the received packets and broadcasts it with the rate $\min \left\{C_{s r_{k}}, C_{d r_{k}}\right\}$ so that both $s$ and $d$ can decode it. Although the 2-step-XOR scheme achieves larger sum-rate than 3-step-XOR from the information theoretical perspective [9], it has no explicit rate expression, requires tight synchronization, and is more difficult to be applied to existing networks. Thus, we only consider the 3 -step-XOR scheme here. Nevertheless, the proposed JSRS algorithm in Section III can be extended to 2-step-XOR easily.

For 3-step-XOR, the total time of one round of packet exchange between nodes $s$ and $d$ through relay $k$ is given by

$$
t_{k}^{2 w}=\frac{1}{C_{s r_{k}}}+\frac{1}{C_{d r_{k}}}+\frac{1}{\min \left\{C_{s r_{k}}, C_{d r_{k}}\right\}} .
$$

As a result, the relay with the minimum $t_{k}^{2 w}$ should be selected.

\section{Joint Scheduling ANd Relay SELECTION}

In this section, we propose the joint scheduling and relay selection algorithms (JSRS) with buffering for both one-way and two-way relay networks, and discuss how to realize JSRS in a distributed way.

\section{A. One-Way Relay Networks}

In one-way relay networks, the packets are transmitted from the source node to the destination with the help from relay nodes, and there is a buffer $B_{k}^{s}$ at each relay $k$. The link space for transmission scheduling is $\mathcal{L}=$ $\left\{l_{s r_{1}}, l_{s r_{2}}, \ldots, l_{s r_{K}}, l_{r_{1} d}, l_{r_{2} d}, \ldots, l_{r_{K} d}\right\}$. 
We assume that there are always packets waiting for transmission at the source node. Note that after the system enters into a steady state, where the buffers at all the relay nodes are non-empty and finite, the long-term throughput of the system can be maximized by scheduling the link in $\mathcal{L}$ with the maximum rate for transmission at each time slot.

In practice, the buffer of each relay is empty at the very beginning of the transmission. During the transmission, it is possible that the buffer of the relay with the maximum rate $C_{r_{k} d}$ to the destination among all $K$ relay nodes is empty, while the buffer of the relay with the maximum rate $C_{s r_{k}}$ is full already and cannot accept any more packet. Taking these practical issues into account, we should consider the following two principles for joint scheduling and relay selection.

- When the buffer of relay $k$ is full, the source node should not transmit packets to it until it sends out some packets and has space for new ones.

- When the buffer is empty, the relay node should not transmit. When the queue size becomes large, the relay should have a high priority to transmit even its rate to the destination is not the maximum. Or the buffer overflow may happen.

Given the above principles, it is difficult to rigorously formulate the joint scheduling and relay selection problem for throughput maximization. Thus we propose a heuristic algorithm, which works as follows.

Let us first define a factor to indicate the queuing status of the buffer at each relay $k$, for $k=1, \ldots, K$, as follows:

$$
\alpha_{k}=1-I\left(Q_{k}^{s}\right) \frac{Q_{k}^{s}}{B_{k}^{s}},
$$

where $B_{k}^{s}$ is the buffer size of relay node $k$, and $Q_{k}^{s}$ is the queue size, or equivalently the number of packets in the buffer $B_{k}^{s}$. Here, $I(\cdot)$ is an indication function defined as follows.

$$
I(x)= \begin{cases}1, & \text { if } x>0, \\ -\infty, & \text { if } x==0 .\end{cases}
$$

Then we scale the transmission rate of each relay to the destination by the queuing factor $\alpha_{k}$ as, while keeping the rate of the source to each relay unchanged.

$$
C_{r_{k} d}^{\prime}=\frac{C_{r_{k} d}}{\alpha_{k}} .
$$

The rate space after scaling $\mathcal{C}=\left\{C_{s r_{1}}, C_{s r_{2}}, \ldots, C_{s r_{K}}, C_{r_{1} d}^{\prime}\right.$, $\left.C_{r_{2} d}^{\prime}, \ldots, C_{r_{K} d}^{\prime}\right\}$ is now treated as a cross-layer relay selection metric. Our proposed JSRS algorithm is then to select the link from the link space $\mathcal{L}$ with the maximum rate in $\mathcal{C}$ for transmission. It can be seen that when the queue size $Q_{k}^{s}$ of relay $k$ increases, the rate $C_{r_{k} d}^{\prime}$ also increases and relay $k$ obtains higher priority to transmit. When the buffer of relay $k$ is full, the corresponding $C_{r_{k} d}^{\prime}$ approaches infinity and, thus, relay $k$ would obtain the highest priority to transmit.

\section{B. Two-Way Relay Networks}

In two-way relay networks, the aim of the joint scheduling and relay selection is to maximize the total throughput, subject to the condition that the throughput of each path is equal to each other. This can be represented as

$$
\begin{aligned}
& \max T_{s r} \\
& \text { s.t. } T_{s r}=T_{d r}=T_{r},
\end{aligned}
$$

where $T_{s r}, T_{d r}, T_{r}$ denote the throughput from node $s$ to the relays, the throughput from node $d$ to the relays, and that from the relays to nodes $s$ and $d$, respectively.

Buffer-empty and buffer-overflow issues should also be considered in two-way relay networks. In addition, since each transmitting relay should take one packet from each of its two buffers to perform XOR, it must ensure that neither of the two buffers are empty before being scheduled .

Thus, we should consider following three principles in JSRS for two-way relay networks:

- When $B_{k}^{s}$ is full, the node $s$ should not transmit packets to relay $k$ until it sends out some packets. Likewise, the node $d$ should not transmit to relay $k$ if $B_{k}^{d}$ is full.

- When either $B_{k}^{s}$ or $B_{k}^{d}$ is empty, the relay $k$ should not be scheduled. When either the queue size $Q_{k}^{s}$ or $Q_{k}^{d}$ approaches the size of the buffer, the relay $k$ should have a high priority to transmit.

- If $Q_{k}^{s}<<Q_{k}^{d}$, the node $s$ should transmit with high priority, and vice versa.

Given the above principles, we now introduce our proposed JSRS algorithm in the following. First, define three factors $\beta_{k}$, $\gamma_{s_{k}}$ and $\gamma_{d_{k}}$ as:

$$
\begin{aligned}
\beta_{k} & =1-I\left(Q_{k, \min }\right) \frac{Q_{k, \max }}{\max \left(B_{k}^{s}, B_{k}^{d}\right)}, \\
\gamma_{s_{k}} & =1+I_{2}\left(Q_{k}^{s}, B_{k}^{s}\right) \frac{Q_{k}^{s}-Q_{k}^{d}}{\min \left(B_{k}^{s}, B_{k}^{d}\right)}, \\
\gamma_{d_{k}} & =1+I_{2}\left(Q_{k}^{d}, B_{k}^{d}\right) \frac{Q_{k}^{d}-Q_{k}^{s}}{\min \left(B_{k}^{s}, B_{k}^{d}\right)},
\end{aligned}
$$

where $Q_{k, \max }=\max \left(Q_{k}^{s}, Q_{k}^{d}\right), Q_{k, \min }=\min \left(Q_{k}^{s}, Q_{k}^{d}\right), I(\cdot)$ is given in (5) and $I_{2}(Q, B)$ is defined as

$$
I_{2}(Q, B)= \begin{cases}1, & \text { if } Q<B, \\ \infty, & \text { if } Q=B .\end{cases}
$$

From the definition (8), $\beta_{k}$ indicates the buffer status at relay $k$. If $Q_{k, \min }>0, \beta_{k}$ is an increasing function of $Q_{k, \max }$. From the definition (9), $\gamma_{s_{k}}$ characterizes the fairness between $Q_{k}^{s}$ and $Q_{k}^{d}$ at relay node $k$ from the perspective of node $s$. If $\gamma_{s_{k}} \leq 1$, there are more packets from node $d$ than that from node $s$ at relay node $k$. If $1<\gamma_{s_{k}} \leq 2, Q_{s_{k}}$ is larger $Q_{d_{k}}$. If $\gamma_{s_{k}}=\infty$, the buffer $B_{k}^{s}$ is full, and the node $s$ should not transmit in this time slot. Likewise, the fairness between $Q_{k}^{d}$ and $Q_{k}^{s}$ at relay node $k$ from the perspective of node $d$ is characterized by $\gamma_{d_{k}}$ as defined in (10).

After introducing the above three factors, the set of the scaled rates on all the possible links becomes:

$$
\begin{aligned}
\mathcal{C}=\{ & \min \left(C_{s r_{1}}, C_{d r_{1}}\right) / \beta_{1}, \min \left(C_{s r_{2}}, C_{d r_{2}}\right) / \beta_{2}, \\
& \ldots, \min \left(C_{s r_{K}}, C_{d r_{K}}\right) / \beta_{K}, \\
& C_{s r_{1}} / \gamma_{s_{1}}, C_{s r_{2}} / \gamma_{s_{2}}, \ldots, C_{s r_{K}} / \gamma_{s_{K}}, \\
& \left.C_{d r_{1}} / \gamma_{d_{1}}, C_{d r_{2}} / \gamma_{d_{2}}, \ldots, C_{d r_{K}} / \gamma_{d_{K}}\right\}
\end{aligned}
$$


The set $\mathcal{C}$ in (12) is now treated as the cross-layer relay selection metrics. Then our proposed JSRS algorithm schedules the link with the maximum rate in $\mathcal{C}$ in each time slot. By using the queuing factor $\beta_{k}$, the relay node with the larger queue size obtains higher priority for transmission if neither of its buffers is empty. If one buffer is empty, the scaled rate of relay $k$ for broadcasting becomes small enough and hence will not be scheduled. By using the fairness factors $\gamma_{s_{k}}$ and $\gamma_{d_{k}}$, the relay with more severe unfairness obtains higher priority to receive packets from node $s$ or node $d$.

\section{Discussion on Distributed Realization}

The two JSRS algorithms proposed above can be realized in a distributed way similar to that in [5]. For the ease of presentation, the distributed relay selection for one-way relay networks without buffering in [5] is briefly described here. The source and destination nodes firstly handshake with some short packets, such as request-to-send (RTS) and clear-to-send (CTS) packets as in IEEE 802.11 based ad hoc networks, through which each relay node estimates its channel conditions associated with both the source and the destination. Note that the exchange of RTS and CTS packets between the source and the destination is reasonable, for the transmission rates of them is relatively slow. Then each relay computes its overall performance metric, such as the end-to-end transmission time in (2), and starts a timer with the delay proportional to it. The timer of the relay with the minimum transmission time will timeout first and the relay sends a short packet to inform the source. Then the source will start transmitting to that relay.

For JSRS in one-way relay networks, the handshaking between the source and the destination is still needed for the relay nodes to obtain the channel state information. After the handshaking, each relay computes the cross-layer metrics $C_{s r_{k}}$ and $C_{r_{k} d}^{\prime}$ according to its buffer status and channel conditions, and starts a timer with the delay inversely proportional to $\max \left(C_{s r_{k}}, C_{r_{k} d}^{\prime}\right)$. Note that, unlike the traditional relay selection, when the timer of a relay, say relay $k^{*}$, counts down to zero firstly, two different cases need to be considered. If the maximum rate of relay $k^{*}$ is $C_{s r_{k}^{*}}$, then relay $k^{*}$ sends a short packet to the source node to trigger the transmission at the source node. Otherwise, if the maximum rate is $C_{r_{k} d}^{\prime}$, the relay will send the packet in its queue directly in the following time slot to the destination.

For JSRS in the two-way relay networks, the algorithm is similar. After timeout occurs, the relay with the maximum $\max \left\{\min \left(C_{r_{k} s}, C_{r_{k} d}\right) / \beta_{k}, C_{s r_{k}} / \gamma_{s_{k}}, C_{d r_{k}} / \gamma_{d_{k}}\right\}$ sends an informing packet to node $s$ or node $d$, if the maximum rate is achieved at $C_{s r_{k}} / \gamma_{s_{k}}$ or $C_{d r_{k}} / \gamma_{d_{k}}$. Or the relay node will send the XORed packets to nodes $s$ and $d$ directly.

Through the timer scheme, we can realize the joint scheduling and relay selection (JSRS) in a distributed way. The derivation of the collision probability and the analysis of the theoretical performance are left as the future work.

\section{Simulation Results}

In this section, we evaluate the performance of the proposed JSRS compared with fixed time division (FTD) scheme for both one-way and two-way relay networks. The effects of average SNR, numbers of relay nodes, and buffer sizes are studied. In all the simulation, the variance of the log-normal shadowing is set to be $5 \mathrm{~dB}$.

\section{A. Throughput vs. Number of Relays}

The throughput comparison between JSRS and FTD at different number of relay nodes is given in Figure 2. Three average SNR values $-10 \mathrm{~dB}, 0 \mathrm{~dB}$, and $10 \mathrm{~dB}$ are considered. As shown in Figure 2, in both one-way and two-way relay networks, the throughput gain of JSRS over FTD is significant at all number of relays. In specific, as shown in Figure 2(a), for one-way relay networks with five relay nodes, the average throughput gains in bits $/ \mathrm{sec} / \mathrm{Hz}$ are $0.04,0.27$, and 0.57 when $\gamma_{0}=-10 \mathrm{~dB}, 0 \mathrm{~dB}$, and $10 \mathrm{~dB}$, respectively. As shown in Figure 2(b), for two-way relay networks with five relay nodes, the average throughput gains in bits/sec/Hz are $0.05,0.35$, and 0.82 when $\gamma_{0}=-10 \mathrm{~dB}, 0 \mathrm{~dB}$, and $10 \mathrm{~dB}$, respectively. These gains are expected as JSRS can achieve the macro-diversity gain brought by relay nodes but also the multi-user diversity.

From the figure it is also seen that the throughput of both JSRS and FTD increases as the number of relay nodes. The throughput with two relay nodes is significantly larger than that with only one relay, while the throughput increasing rate decreases as the number of relay increases. This agrees with the diminishing effect of diversity gain.

\section{B. Throughput Improvement Ratio vs. Buffer Size}

The buffer sizes of each relay are fixed to be $B=500$ and $B=1000$ here. Figure 3 shows the throughput improvement ratios of JSRS over FTD with the two buffer sizes at different average SNR. Generally, the throughput improvement ratio decreases as the average SNR increases, for the relative difference between the rate of each link decreases as the channel gain increases. For one-way relay networks as shown in Figure 3(a), the throughput improvement ratio with larger buffer sizes is larger than that with smaller values. When the average SNR is large, the throughput improvement ratio with one relay is larger than that with four relay nodes. However, for $\gamma_{0}<-8 \mathrm{~dB}$ with $B=1000$, and $\gamma_{0}<0 \mathrm{~dB}$ with $B=500$, the throughput improvement ratio with one relay is smaller than that with four relay. It indicates that JSRS utilizes the space diversity gain with relatively small channel gain better than that with larger channel gain. Note that the absolute rates of both JSRS and FTD with small average SNR is small, although the ratio is large.

For the two-way relay networks as shown in Figure 3(b), the throughput improvement ratios with larger buffer size are a bit larger than that with smaller buffer size. The ratio with one relay is much larger than that with four relay nodes, since the absolute throughput of both JSRS and FTD with four relay nodes is larger than that with only one relay, while the gap is not increased as explained in Section IV-A. 


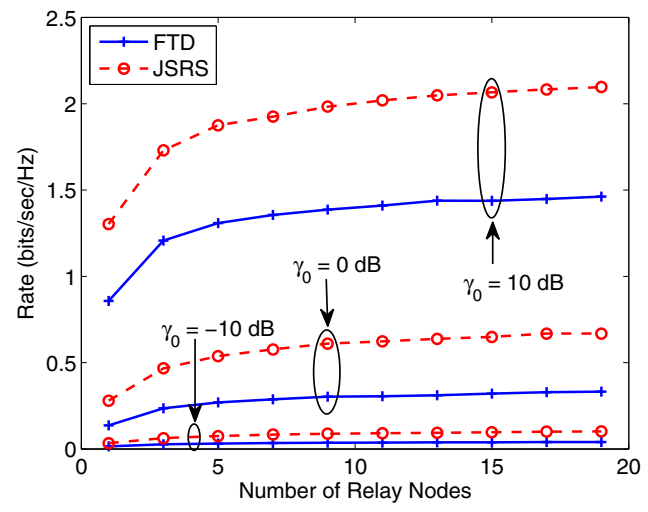

(a) One-way relay networks

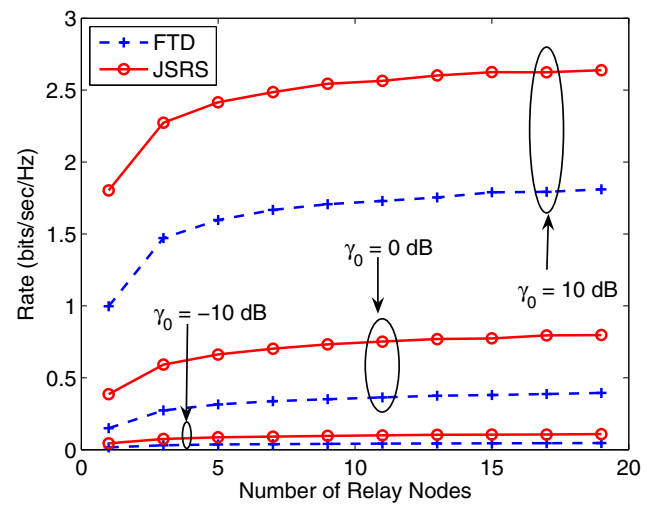

(b) Two-way relay networks

Fig. 2. Throughput of JSRS and FTD with different number of relay nodes in both one-way and two-way relay networks.

The results in Figure 3 show that the performance of our JSRS algorithm is mainly influenced by the channel gain and the number of relay nodes, while the buffer size has minor impact on it.

\section{CONCLUSiON}

It is shown in this paper that the joint scheduling and relay selection with buffering can improve the rate of both one-way and two-way relay networks. We introduced the optimal relay selection metrics for fixed time division (FTD) scheme, and then proposed our joint scheduling and relay selection algorithms JSRS for both one-way and two-way relay networks with the novel cross-layer relay selection metrics taking into account both instantaneous channel conditions and queuing status. We also discussed that JSRS can be realized in a distributed way without explicit coordination among the network nodes. Simulation results show that the throughput of JSRS is significantly larger than that of FTD. The results also show that the buffer sizes only have a minor influence on the performance of JSRS. In the future work, we will investigate the delay performance and power control.

\section{REFERENCES}

[1] A. Nosratinia, T.E. Hunter, and A. Hedayat, "Cooperative communication in wireless networks," IEEE Commun. Magazine, vol. 42, no. 10, pp. $74-80$, Oct. 2004.

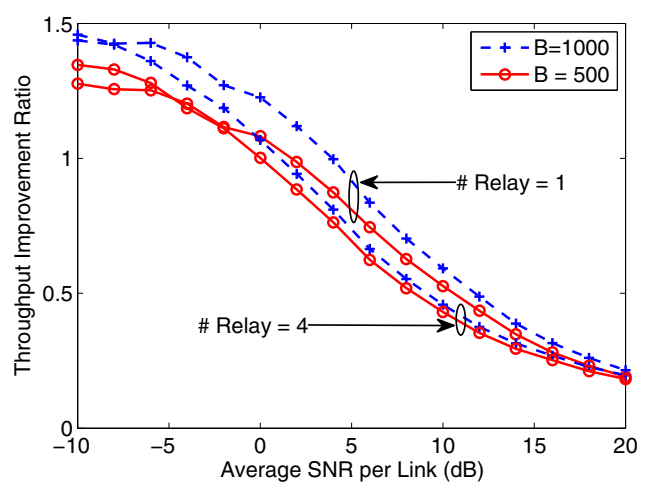

(a) One-way relay networks

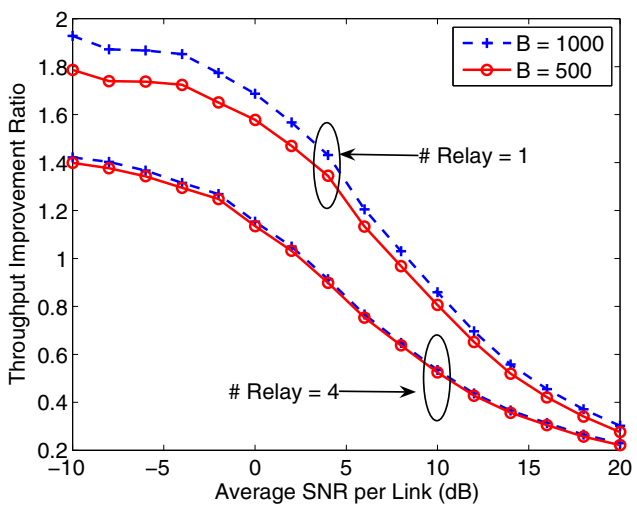

(b) Two-way relay networks

Fig. 3. Throughput improvement ratio with different buffer sizes in both one-way and two-way relay networks.

[2] Y. Zhao, R. Adve, and T. J. Lim, "Improving amplify-and-forward relay networks: Optimal power allocation versus selection," IEEE Trans. on Wireless Commun., vol. 6, no. 8, Aug. 2007.

[3] E. Beres, and R. Adve, "On selection cooperation in distributed networks," in Proc. CISS, Mar. 2006.

[4] Y. Li, B. Vucetic, Z. Chen, and J. yuan, "An improved relay selection scheme with hybrid relaying protocols," in IEEE Proc. GLOBECOM, Nov. 2007.

[5] A. Bletsas, H. Shin, and M. Z. Win, "Cooperative communications with outage-optimal opportunistic relaying," IEEE Trans. on Wireless Commun., vol. 6, no. 9, pp. 3450-3460, Sep. 2007.

[6] A. Bletsas, A. Khisti, D. P. Reed, and A.Lippman, "A simple cooperative diversity mothod based on network path selection," IEEE JSAC, vol. 24, no. 3, pp. 659-672, Mar. 2006.

[7] B. Rankov, and A. Wittneben, "Spectral efficient signaling for halfduplex relay channels," IEEE JSAC, vol. 25, no. 2, Feb. 2007.

[8] P. Larsson, N. Johansson, and K. E. Sunell, "Coded bidirectional relaying," in IEEE Proc. VTC-Spring, vol. 2, May. 2006, pp. 851-855.

[9] P. Popovski, and H. Yomo, "Physical network coding in two-way wireless relay channels," in IEEE Proc. ICC, Jun. 2007, pp. 707-712.

[10] P. Popovski, and H. Yom, "Bidirectional amplification of throughput in a wireless multihop networks," in IEEE Proc. VTC-Spring, May. 2006, pp. 588-593.

[11] T. J. Oechtering, and H. Boche, "Bidirectional regenerative half-duplex relaying using relay selection," IEEE Trans. on Wireless Commun., vol. 7, no. 5, May. 2008.

[12] Y. Wu, P. A. Chou, and S. Y. Kung, "Information exchange in wireless networks with network coding and physical-layer broadcast," in Proc. 2005 Conference on Information Sciences and Systems, Mar. 2005.

[13] O. Oyman and S. Sandhu, "Non-ergodic power-bandwidth tradeoff in linear multi-hop networks," in Proc. ISIT, Jul. 2006. 